\title{
Resonantly enhanced Faraday rotation in a microcoil current sensor
}

\author{
G. Y. Chen, T. Lee, R. Ismaeel, G. Brambilla, and T. P. Newson
}

\begin{abstract}
A proof-of-principle experimental demonstration with theoretical modeling is presented for resonantly enhanced Faraday rotation in a microcoil current sensor. The recirculation of resonant light within the coil gives rise to accumulated polarization rotation and thus improved responsivity. According to simulations, microcoil resonators with sharper resonances could offer significantly larger enhancements. This new type of current sensor has the potential to be ultra-fast, compact and low-cost.
\end{abstract}

Index Terms-Current sensor, Faraday Effect, Microcoil resonator.

\section{INTRODUCTION}

$\mathrm{F}$ iber-optic current sensors that exploit the Faraday Effect [1-3] have attracted a great deal of interest due to their wide dynamic range, robustness and remote sensing capability. However, those based on conventional optical fiber have been relatively bulky due to the requirement of satisfying minimum fiber bend radius criteria. Recently, a new approach to current sensing using optical fiber micro-wire (OFM) in the configuration of a microcoil (MC) has been proposed and demonstrated [4]. It featured high compactness and configurability. In this Letter, we present a theoretical and experimental demonstration of resonantly enhanced Faraday rotation using microcoil resonators (MCRs) and the potential to attain higher responsivity with sharper resonances. The resonant polarization component has a large phase change owing to the cavity resonance. This type of Faraday rotation enhancement used for current sensing has several advantages compared to other reported methods [5,6]. Firstly, MCRs are highly compact devices that can be deployed in places with very limited space. Secondly, it offers the possibility of performing non-contact measurements, making it an attractive method for probing miniature electrical components. Lastly, it has negligible coupling losses to its two fiber pigtails due to the adiabatic transition along the taper.

Manuscript received December 14, 2011; revised Janurary xx, 2012; accepted February xx, 2012. Date of publication March xx, 2012; date of current version March xx, 2012.

G. Y. Chen, T. Lee, R. Ismaeel, G. Brambilla, and T. P. Newson are with the Optoelectronics Research Centre, University of Southampton, Southampton, SO17 1BJ UK (e-mail: gyc1g09@orc.soton.ac.uk; t1305@orc.soton.ac.uk; rmni1g10@orc.soton.ac.uk; gb2@orc.soton.ac.uk; tpn@orc.soton.ac.uk).

\section{PRINCIPLE}

An OFM [7] is a dielectric optical waveguide with a diameter of the order of 1 micron. The fundamental mode of an OFM is well-confined due to the large refractive index difference between silica and the surrounding medium. A MC is a helical arrangement of OFM with no coupling between adjacent turns. Unlike the MC, the MCR has a smaller pitch that enables coupling between adjacent turns. The Biot-Savart law describes the magnetic field $(B)$ as a function of distance from a current-carrying conductor. For a MCR coiled around such a conductor, the light experiences a magnetic field which can be expressed by:

$$
B=\left(\mu_{0} \mu_{r} I\right) /(2 \pi r)
$$

where $\mu_{0}$ is the magnetic permeability, $\mu_{r}$ is the relative permeability of the fiber, $I$ is the current and $r$ is the radius of the fiber coil. The Faraday rotation $\left(\theta_{0}\right)$ of the plane of linearly polarized light for a single turn is given by:

$$
\theta_{0}=V B(2 \pi r)=\tau(2 \pi r)=\mu_{0} \mu_{r} V I
$$

where $V$ is the Verdet constant of the silica MCR and $\theta_{0}$ is expressed in terms of $\tau$, which is defined as the angle of rotation per unit length.

To model the Faraday Effect in a MCR, the underlying coupled mode equations must be modified by incorporating a $\tau$ term to account for the polarization rotation [8]. The $\Delta \beta$ term models the bend birefringence and $\alpha$ describes the optical loss. The Berry phase and polarization dependency of the coupling factors have been neglected. For the $j^{\text {th }}$ turn between $j=2$. $(N-1)$, the changes in the electric field amplitude components $A_{x}^{j}$ and $A_{y}^{j}$ aligned with the normal and binormal axes are expressed by:

$$
\begin{aligned}
& \frac{d A_{x}^{j}}{d s}=i \kappa\left(A_{x}^{j-1}+A_{x}^{j+1}\right)+i \Delta \beta A_{x}^{j}-\alpha A_{x}^{j}-\tau A_{y}^{j} \\
& \frac{d A_{y}^{j}}{d s}=i \kappa\left(A_{y}^{j-1}+A_{y}^{j+1}\right)-\alpha A_{y}^{j}+\tau A_{x}^{j}
\end{aligned}
$$

where $N$ is the total number of turns, $s$ is the curvilinear distance along the fiber axis, and $\kappa$ is the coupling coefficient between adjacent turns. For the first and last turn, Eq. 3 and Eq. 4 must be modified such that the light only couples to the second and penultimate turn respectively. The ordinary differential equations are then solved numerically under the boundary conditions imposed by field continuity [8]. 

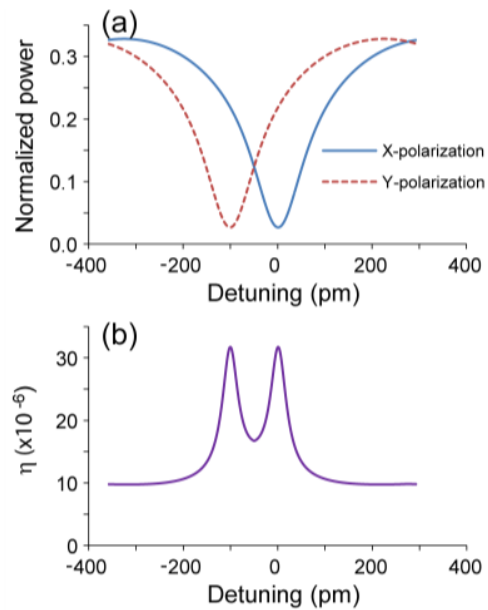

Fig. 1. (a) Simulated transmission spectra of $\operatorname{MCR}(\alpha=3 \mathrm{~dB} / \mathrm{cm}, N=3, r=0.5$ $\left.\mathrm{mm}, \mu_{0}=1.256 \times 10^{-6}, \mu_{r}=1, V=0.54 \mathrm{rad} / \mathrm{T} . \mathrm{m}, I=7.9 \mathrm{~A}, \kappa=5523 / \mathrm{m}\right)$, and (c) the relationship between resonant ER and enhancement factor $N$.

To begin with, we study the output spectra of a birefringent MCR in Fig. 1(a) with $\Delta n=7.69 \times 10^{-5}$ and extinction ratio (ER) of $10.9 \mathrm{~dB}$. Assuming that the initial power is equal in both axes, it can be shown from Eq. 6 that $\eta=\Delta P / P$ is a figure of merit for Faraday rotation based on the percentage change in power $(P)$ for a particular polarization. Fig. 1(b) shows the Faraday rotation enhancement factor comparing on- and off-resonance to be: $N=\eta_{\text {res }} / \eta_{\text {off-res }}=3.25$. It is clear from Fig. 1 (c) that MCRs with higher Q-factors provide larger enhancements due to the recirculation of resonant light within the coil, and hence experiences an effective optical length longer than the physical length of the coil. Yet, the increased transit time required by resonant light to propagate through the MCR will reduce the upper limit of the device bandwidth.

\section{EXPERIMENT}

To experimentally confirm the simulations, a $2 \mu \mathrm{m}$ diameter, $10 \mathrm{~mm}$ long OFM was manufactured from a telecom single-mode fiber (Corning SMF-28) using the micro-heater brushing technique [9]. A thin layer of polymer (EFIRON $\mathrm{UV}-373, n=1.37$ ) was deposited on a $1 \mathrm{~mm}$ diameter copper wire before coiling the OFM. U.V. curing of the polymer provided robust packaging. During the fabrication, the MCR transmission spectrum was measured in situ to ensure low optical loss. The resulting MCR had 3 turns with a pitch of a few microns between each winding to permit coupling between adjacent turns.

As shown in Fig. 2, linearly polarized light is launched into polarization-maintaining (PM) fiber from a tunable laser source (TLS). The $45^{\circ}$ splice produces $\mathrm{x}$ and $\mathrm{y}$-polarized light of equal power, which propagate through the polarization-maintaining coupler (PMC) to the MCR via a polarization controller (PC). A signal generator driving a transformer induces AC current along the copper wire that translates into a varying magnetic field. When no current is applied, the light propagating along each axis will be reflected back via the other axis by the Faraday rotator mirror (FRM), which is used to compensate for state of polarization (SOP) fluctuations along the sensing fiber. The

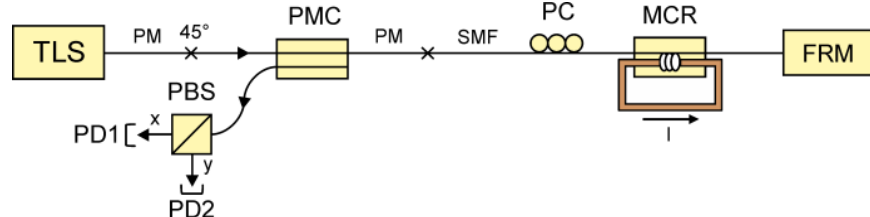

Fig. 2. Optical diagram of current interrogation system based on balanced detection.

light re-entering the $\mathrm{PM}$ fiber translates to an intensity modulation based on the incident angle. At the polarization beam-splitter (PBS), the $\mathrm{x}$ and $\mathrm{y}$-polarized light are separated into the ports of a balanced detector [10], which eliminates common noise and amplifies the difference between the two signals. An AC-coupled oscilloscope was used to record 1000 data points per measurement, with an averaging of 32 .

The sinusoidal current creates a varying magnetic field which modulates the angle of Faraday rotation $(\theta)$.

$$
\theta=\theta_{0} \sin (\omega t+\phi)
$$

For PD1 and PD2, the corresponding $\mathrm{x}$ and $\mathrm{y}$-polarized electric field amplitudes $\left(A_{\text {out }}\right)$ can be derived from the product of the Jones matrices for the non-reciprocal Faraday rotations and the Faraday rotator mirror.

$$
\left(\begin{array}{l}
A_{x}^{\text {out }} \\
A_{y}^{\text {out }}
\end{array}\right)=\left(\begin{array}{cc}
\sin 2 \theta & \cos 2 \theta \\
-\cos 2 \theta & -\sin 2 \theta
\end{array}\right) \cdot\left(\begin{array}{c}
A_{x}^{\text {in }} \\
A_{y}^{\text {in }}
\end{array}\right)
$$

The function of the balanced detector is given by:

$$
V^{\text {out }} \propto\left(\left|A_{y}^{\text {out }}\right|^{2}-\left|A_{x}^{\text {out }}\right|^{2}\right) /\left(\left|A_{x}^{\text {out }}\right|^{2}+\left|A_{y}^{\text {out }}\right|^{2}\right)
$$

The output voltage $\left(V_{\text {out }}\right.$ ) for a $45^{\circ}$ splice angle is given by:

$$
V_{45^{\circ}}^{\text {out }} \propto \sin 4 \theta
$$

Alternatively, expanded in terms of Bessel functions of the first kind:

$$
V_{45^{\circ}}^{\text {out }} \propto 2 \sum_{n=1,3, . .} J_{n}\left(4 \theta_{0}\right) \sin (n(\omega t+\phi))
$$

Similarly, for a $0^{\circ}$ splice angle:

$$
V_{0^{\circ}}^{\text {out }} \propto \cos 4 \theta
$$

Likewise, in terms of Bessel functions of the first kind:

$$
V_{0^{\circ}}^{\text {out }} \propto J_{0}\left(4 \theta_{0}\right)+2 \sum_{n=2,4, . .} J_{n}\left(4 \theta_{0}\right) \cos (n(\omega t+\phi))
$$

Eq. 9 shows that a $45^{\circ}$ splice produces a signal predominately of fundamental frequency, and Eq. 11 shows that a $0^{\circ}$ splice results in even harmonics.

Fig. 3 shows the double-pass transmission spectra of the fabricated MCR with a Q-factor of 17000 , resonant ER of 10.9 $\mathrm{dB}$, birefringence-induced resonance separation of $100 \mathrm{pm}$, and loss of $3 \mathrm{~dB}$. The wavelengths at which the Faraday Effect was investigated are $\lambda_{0}=1550.015 \mathrm{~nm}, \lambda_{M}=1550.222 \mathrm{~nm}$ and $\lambda_{R}=1550.270 \mathrm{~nm}$; which correspond to off-resonance, midway-to-resonance, and on-resonance respectively. 


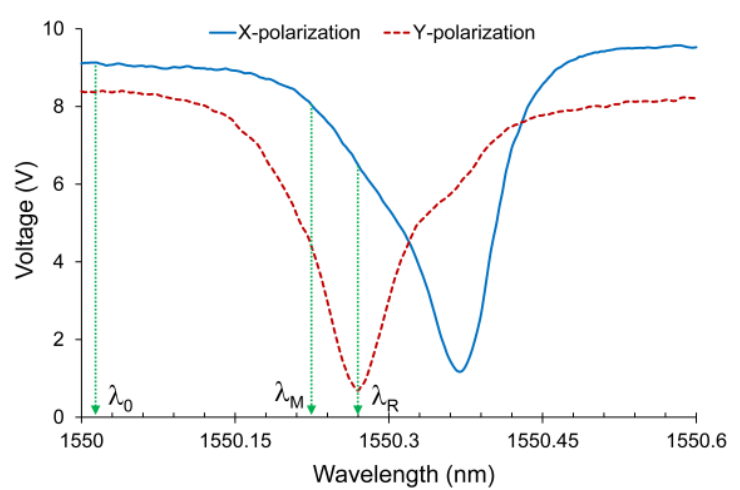

Fig. 3. Measured double-pass transmission spectra of the MCR for $\mathrm{x}$ and y-polarized light, with no current applied.

The measured signal was dominated by the second harmonic of the current signal. This can be attributed to the MCR having significantly higher loss in one axis. Although the FRM would normally compensate for the polarization dependent loss, the $\mathrm{x}$ and y-polarized light do not re-enter the MCR at $90^{\circ}$ to the incident angle in the presence of Faraday rotation. Hence, under such circumstances the device behaves as a $0^{\circ}$ splice.

Fig. 4 compares the optical signals at the selected wavelengths. An enhancement of $N=3.1$ was calculated for $\lambda_{R}$ by using Eq. 10, which is close to the simulated value of $N=$ 3.25 from Fig. 1. This difference is most likely caused by the non-ideal resonance spectra obtained in the experiment. Fig. 5 shows a linear responsivity of $4.4 \mu \mathrm{rad} / \mathrm{A}$ that is comparable but lower than the expected value of $6.3 \mu \mathrm{rad} / \mathrm{A}$, derived from the experimentally obtained value for $N$ and Eq. 2 . This disparity is due to linear birefringence arising from micro-bending, which reduces the rotation of linearly polarized light.

Unlike in our experiment, where the passively damped optical bench minimized the effect of vibrations, in a real measurement environment unsuppressed mechanical vibrations may influence the geometric stability of the MCR at the current signal frequency of $30 \mathrm{~Hz}$ and the corresponding optical second-harmonic frequency of $60 \mathrm{~Hz}$, thus causing errors in the measured Faraday rotation.
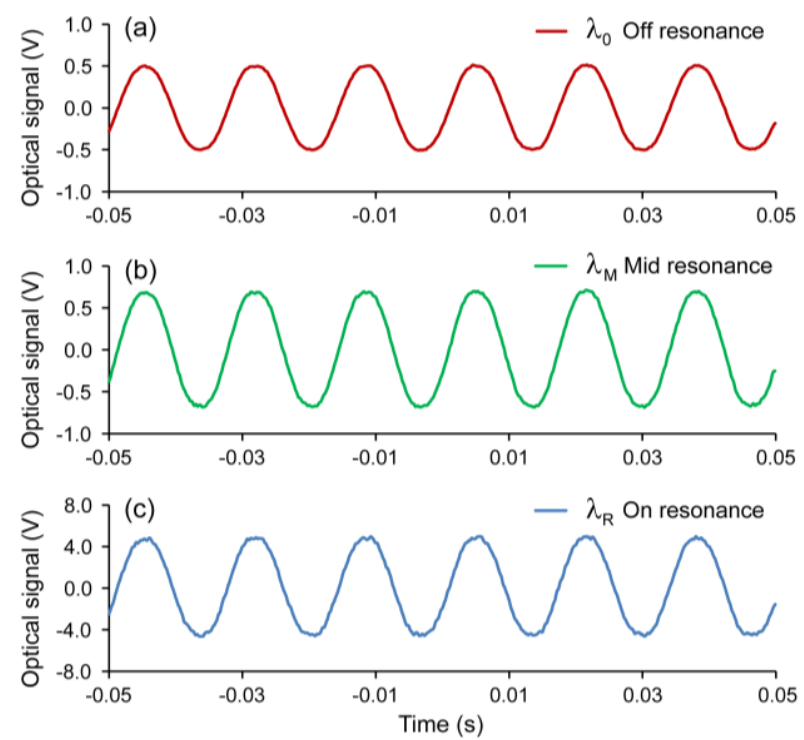

Fig. 4. Optical signal at (a) $\lambda_{0}$ (b) $\lambda_{M}$ and (c) $\lambda_{R}$ in response to current signal modulated at $7.9 \mathrm{~A}, 30 \mathrm{~Hz}$.

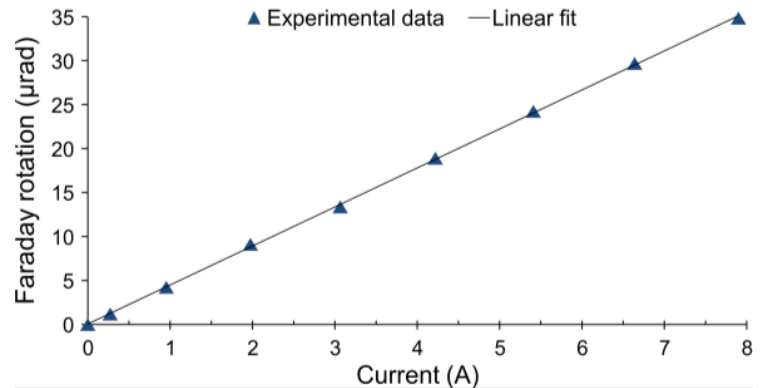

Fig. 5. Optical responsivity at $\lambda_{R}$ to current signal modulated at $7.9 \mathrm{~A}, 30 \mathrm{~Hz}$.

MCR-based current sensors are even more compact than the MC type, since they only require a few turns as their responsivity depend more on proximity from critical coupling and detuning from resonance than the physical length. On the other hand, to attain equivalent responsivity with MCs would require a much larger number of turns using longer tapers, which may not be feasible using conventional tapering rigs.

\section{CONCLUSION}

In conclusion, we theoretically predicted that Faraday rotation can be enhanced by the resonant cavity of the MCR due to the fact that the Faraday Effect is non-reciprocal in nature. As a proof-of-principle, a MCR with a Q-factor of 17000 demonstrated a factor of 3.1 increase in Faraday rotation between on- and off-resonance. According to simulations, sharper resonances will lead to significantly larger enhancements and thus considerably improving the responsivity of the sensor. MCR-based current sensors have the potential to be ultra-fast, compact and low-cost.

\section{REFERENCES}

[1] G. W. Day, D. N. Payne, A. J. Barlow, and J. J. Ramskov-Hansent, "Faraday rotation in coiled, monomode optical fibers: isolators, filters, and magnetic sensors", Opt. Lett., vol. 7, no. 5, pp. 238-240, May 1982.

[2] J. Blake, P. Tantaswadi, R. T. de Carvalho, "In-line Sagnac interferometer current sensor", IEEE Trans. Power Del., vol. 11, no. 1, pp. 116-121, Jan. 1996.

[3] S. Kramer, F. P. Leon, Y. M. Hernandez, B. Lewke, "Fiber optic current sensors for high current surge measurements", SENSOR Conf. Proc. II, B7.2, pp. 135-139, 2007.

[4] M. Belal, Z. Song, Y. Jung, G. Brambilla, and T. P. Newson, "Optical fiber microwire current sensor", Opt. Lett., vol. 35, no. 11, pp. 30453047, Sep. 2010.

[5] H. Zhang, Y. Dong, J. Leeson, L. Chen, and X. Bao, "High sensitivity optical fiber current sensor based on polarization diversity and a Faraday rotation mirror cavity", Appl. Opt., vol. 50, no. 6, pp. 924-929 Feb. 2011.

[6] S. M. Hamidi and M. M. Tehranchi, "High transmission enhanced Faraday rotation in coupled resonator magneto-optical waveguides", $J$. Lightwave Technol., vol. 28, no. 19, pp. 2139-2145, Aug. 2010.

[7] G. Brambilla, "Optical fibre nanowires and microwires: a review", $J$. Opt., vol. 12, no. 043001, pp. 1-19, Mar. 2010.

[8] T. Lee, N. G. R. Broderick, and G. Brambilla, "Berry phase magnification in optical microcoil resonators," Opt. Lett., vol. 36, no. 15, pp. 28392841, Jul. 2011.

[9] G. Brambilla, E. Koizumi, X. Feng, and D. J. Richardson, "Compoundglass optical nanowires," Electron. Lett., vol. 41, no. 7, pp. 400-401, Mar. 2005.

[10] R. I. Laming and D. N. Payne, "Electric current sensors employing spun highly birefringent optical fibers", J. Lightwave Technol., vol. 7, no. 12, pp. 2084-2094, Dec. 1989. 